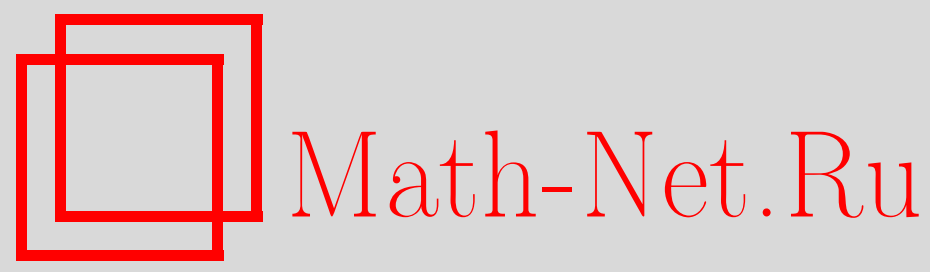

Г. М. Губреев, А. А. Тарасенко, Представимость матрицы де Бранжа в виде произведения Бляшке-Потапова и полнота некоторых семейств функций, Матем. заметки, 2003, том 73, выпуск 6, 841-847

DOI: https://doi.org/10.4213/mzm231

Использование Общероссийского математического портала Math-Net.Ru подразумевает, что вы прочитали и согласны с пользовательским соглашением http://www . mathnet.ru/rus/agreement

Параметры загрузки:

IP : 3.80 .253 .173

26 апреля 2023 г., $16: 47: 57$

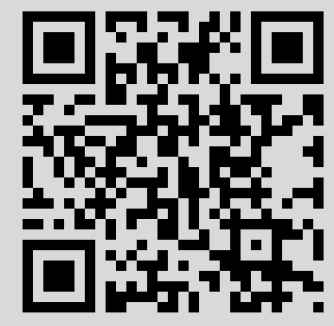




\title{
ПРЕДСТАВИМОСТЬ МАТРИЦЫ ДЕ БРАНЖА \\ В ВИДЕ ПРОИЗВЕДЕНИЯ БЛЯШКЕ-ПОТАПОВА И ПОЛНОТА НЕКОТОРЫХ СЕМЕЙСТВ ФУНКЦИЙ
}

\author{
Г. М. Губреев, А.А. Тарасенко
}

\begin{abstract}
В работе формулируются критерии представимости мероморфных $J$-растягивающих в верхней полуплоскости матриц-функций 2-го порядка (матрицы де Бранжа) в виде левого, правого и двустороннего произведения Бляшке-Потапова. Сформулированы также результаты о спектральной структуре операторов, чьи характеристические матрицы-функции являются матрицами де Бранжа.

Библиограбфия: 15 названий.
\end{abstract}

Пусть $W$ - произвольная мероморфная $J$-растягивающая в верхней полуплоскости $\mathbb{C}_{+}$матрища-функция (м.-ф.), т.е.

$$
W(z) J W^{*}(z) \geqslant J, \quad J^{2}=E, \quad J^{*}=J, \quad J \neq \pm E,
$$

в каждой точке голоморфности $W$. В силу фундаментальной теоремы Потапова [1] м.-ф. $W$ представима в виде

$$
W(z)=\pi(z) \varepsilon(z)=\varepsilon_{1}(z) \pi_{1}(z),
$$

где $\pi, \pi_{1}$ - произведение простейших мероморфных $J$-растягивающих м.-ф. (произведение Бляшке-Потапова), $\varepsilon, \varepsilon_{1}$ - голоморфные в верхней полуплоскости $J$-растягивающие м.-ф., которые изображаются с помошью мультипликативных интегралов. В отличие от дефинитного случая $J= \pm E$ задача о выяснении условий, при которых в представлениях (1) одна из м.-ф. $\varepsilon, \varepsilon_{1}$ (или обе одновременно) тождественно равны единичной матрице $E$, оказалась сложной, причем все трудности этой задачи проявляются уже на м.-ф. второго порядка.

Заметка посвящена критериям представимости важного для приложений класса матриц де Бранжа [2] в виде произведения Бляшке-Потапова. Наш подход основан на простых геометрических рассмотрениях, которые пригодны и в общем случае, а методы доказательств теорем опираются на теорию характеристических м.-ф. несамосопряженных операторов [3], теорию цельх функций класса Картрайт [4], [5] и на свойства семейств рациональных дробей в весовых пространствах [6].

Другой подход к рассматриваемой задаче основан на теории регулярных и строго регулярных $J$-внутренних м.-ф. (см. [7] и список литературы, приведенньй в этой работе). В этих работах интересующие нас критерии явно не формулировались, однако они могут быть получены в рамках изложенной там теории. Сравнению результатов, которые можно получить этими двумя методами, будет посвящена отдельная работа. 
1. Мероморфная во всей комплексной плоскости матрица

$$
W(z)=\left(\begin{array}{ll}
\delta(z) & \gamma(z) \\
\beta(z) & \alpha(z)
\end{array}\right)
$$

назьвается матриией де Бранжа, если

1) $W$ является $J$-растягивающей в $\mathbb{C}_{+}$с матрицей

$$
J=\left(\begin{array}{cc}
0 & i \\
-i & 0
\end{array}\right)
$$

2) почти всюду на вещественной оси

$$
W(x) J W^{*}(x)=J, \quad x \in \mathbb{R}
$$

3) функция $\Phi(z):=(\delta(z) i+\gamma(z)) /(\beta(z) i+\alpha(z))$ голоморфна на $\mathbb{R}$.

В силу общей процедуры отщепления элементарных делителей $[1],[3]$, множители Бляшке-Потапова, отвечающие вещественным полюсам $W$ заведомо входят в м.- $ф . \varepsilon, \varepsilon 1$ из представления (1). Поэтому мы должны предположить, что $W$ голоморфна на $\mathbb{R}$ $u$ совершенна [2], [8], т.е. $\lim _{y \rightarrow \infty} y^{-1} \Phi(i y)=0$. Заметим [8], что если полюсы $W$ не лежат на вещественной оси, то условие 2 ) имеет место всюду на $\mathbb{R}$, а условие 3 ) вьполняется автоматически. Будем считать также, что м.-ф. $W$ нормирована условием

$$
W(0)=E \text {. }
$$

Обозначим через $\Lambda=\left\{\lambda_{n}\right\}_{1}^{\infty}$ множество всех полюсов матрицы де Бранжа $W$, занумерованное в порядке неубьвания модулей. Для упрощения формулировок следующих ниже теорем 1,8 будем предполагать, что все полюсы $W$ простые. Формулировки всех остальных результатов работы остаются в силе и в случае непростых полюсов.

Матрица де Бранжа назьвается правым произведением Бляике-Потапова [1], [3], если имеет место представление

$$
W(z)=\prod_{\lambda_{n} \in \Lambda}^{\rightarrow}\left(E-\frac{i \lambda_{n} z}{\lambda_{n}-z} R_{n}^{*} R_{n} J\right)
$$

где $R_{n}:=\left(r_{n 1}, r_{n 2}\right)$ - последовательность однострочных постоянных матриц, причем $R_{n} J R_{n}^{*}=-2 \operatorname{Im} \lambda_{n}^{-1}, \lambda_{n} \in \Lambda$. Стрелка в (4) указьвает на порядок перемножения элементарных множителей, матрица $J$ определяется равенством (2). Бесконечное произведение (4) сходится равномерно по крайней мере на любом компакте плоскости, который не пересекается с $\Lambda \cup \mathbb{R}$.

Аналогично, м.-ф. $W$ называется левым произведением Бляике-Потапова, если

$$
W(z)=\prod_{\lambda_{n} \in \Lambda}^{\leftarrow}\left(E+\frac{i \lambda_{n} z}{\lambda_{n}-z} L_{n}^{*} L_{n} J\right), \quad L_{n} J L_{n}^{*}=2 \operatorname{Im} \lambda_{n}^{-1}
$$

где $L_{n}:=\left(l_{n 1}, l_{n 2}\right)$ - последовательность постоянных однострочньх матриц. И, наконец, м.-ф. $W$ называется двусторонним произведением Бляике-Потапова, если одновременно имеют место представления (4), (5). 
Каждая матрица де Бранжа (2) допускает вещественное представление [8]

$$
W(z)=\frac{1}{S(z)}\left(\begin{array}{cc}
d(z) & c(z) \\
-b(z) & a(z)
\end{array}\right)
$$

в котором $a, b, c, d$ - целые вешественные функции, причем функция $E(z):=a(z)-b(z) i$ удовлетворяет неравенству

$$
|E(\bar{z})|<|E(z)|, \quad z \in \mathbb{C}_{+}
$$

Поэтому каждая матрица де Бранжа порождает пространство де Бранжа $\mathscr{H}(E)[2]$, состоящее из таких целых функций $F$, что

1) $F / E, F^{*} / E-$ функции ограниченного вида и неположительного среднего типа в $\mathbb{C}_{+}$;

2) $\int_{\mathbb{R}}|F(x) / E(x)|^{2} d x<\infty$.

Здесь использовано обозначение $F^{*}(z):=\overline{F(\bar{z})}$, а средний тип голоморфной в $\mathbb{C}_{+}$функции $f$ вычисляется по формуле

$$
h(f)=\limsup _{y \rightarrow+\infty} \frac{\log |f(i y)|}{y} .
$$

Кроме того, целая функция $S$ из представления (6), обладает такими свойствами:

1) $S / E, S^{*} / E$ суть функции ограниченного вида и неположительного среднего типа в $\mathbb{C}_{+}$;

2) $\int_{\mathbb{R}} \frac{|S(x) / E(x)|^{2}}{1+x^{2}} d x<\infty$

Целые функции $S$, удовлетворяющие этим двум условиям, называются conymcmвyющ,ими пространству $\mathscr{H}(E)$.

Пространство $\mathscr{H}(E)$ является гильбертовым пространством целых функций со скалярным произведением

$$
\langle F, G\rangle:=\int_{\mathbb{R}} F(x) G^{*}(x)|E(x)|^{-2} d x,
$$

а ядро

$$
k(z, \lambda)=\frac{b(z) \overline{a(\lambda)}-a(z) \overline{b(\lambda)}}{\pi(z-\bar{\lambda})}
$$

является воспроизводящим, т.е. $\langle F(x), k(x, \lambda)\rangle=F(\lambda), F \in \mathscr{H}(E), \lambda \in \mathbb{C}$.

2. Итак, мы рассматриваем совершенные матрицы де Бранжа (2), не имеющие полюсов на $\mathbb{R}$. Более того, для упрощения формулировок будем считать, что на вещественной оси нет и ложных полюсов $W$, т.е. $S(x) \neq 0, x \in \mathbb{R}$ (ложных невещественных полюсов быть не может).

В пространстве де Бранжа $\mathscr{H}(E)$, порожденном представлением $(6)$, рассмотрим оператор

$$
(B F)(z):=\frac{F(z) S(0)-S(z) F(0)}{z S(0)}, \quad F \in \mathscr{H}(E) .
$$


Несложные вычисления [9] показывают, что оператор $B-B^{*}$ не более, чем двумерен и характеристическая м.-ф. оператора $B$ равна матрице де Бранжа $W$. Далее, оператор $B$ вполне несамосопряжен, а его собственные векторы совпадают с функциями семейства $\left\{S(z) /\left(z-\lambda_{n}\right): \lambda_{n} \in \Lambda\right\}$. Из теории характеристических м.ф. [3] следует, что представимость $W$ правьп произведением Бляшке-Потапова равносильна полноте этого семейства в пространстве $\mathscr{H}(E)$. Аналогично, $W$ есть левое произведение БляшкеПотапова тогда и только тогда, когда полна в $\mathscr{H}(E)$ система $\left\{k\left(z, \lambda_{n}\right): \lambda_{n} \in \Lambda\right\}$, функции которой совпадают с собственными векторами оператора $B^{*}$.

Переходя в (4), (5) к детерминантам, получим

$$
\alpha(z) \delta(z)-\beta(z) \gamma(z)=\prod_{\lambda_{k} \in \Lambda} \frac{z-\bar{\lambda}_{n}}{z-\lambda_{n}} \frac{\lambda_{n}}{\bar{\lambda}_{n}},
$$

где бесконечное произведение равномерно сходится на любом компакте комплексной плоскости, не содержащем числа $\lambda_{n} \in \Lambda$. Из условия (3) вытекает, что функция $b(z) / z$ целая, а поэтому принадлежит $\mathscr{H}(E)$.

Теорема 1. а) Матрица $W$ тогда и только тогда является правым произведением Бляшке-Потапова, когда имеет место равенство (8) $и$

$$
\frac{b(z)}{z} \in \underset{\mathscr{H}(E)}{\operatorname{clos} \operatorname{span}}\left\{\frac{S(z)}{z-\lambda_{n}}: \lambda_{n} \in \Lambda\right\} .
$$

b) Матрица $W$ тогда и только тогда является левым произведением Бляике-Потапова, когда имеет место равенство (8) и

$$
\frac{b(z)}{z} \in \underset{\mathscr{H}(E)}{\operatorname{clos} \operatorname{span}}\left\{k\left(z, \lambda_{n}\right): \lambda_{n} \in \Lambda\right\} .
$$

Используя стандартные формулы теории приближений, можно сформулировать критерии принадлежности “пробной” функции $b(z) / z$ указанным в формулировке теоремы подпространствам. Получаемые на этом пути критерии (особенно в случае непростых полюсов) довольно громоздки, что затрудняет их применение. Вместе с тем, если соединить теорему 1 с теорией функций класса Картрайт, а также с некоторыми результатами работы [6], то можно получить вполне обозримые формулировки.

Напомним [4], [5], что целая функция экспоненциального типа (ц.ф.э.т.) $\varphi$ принадлежит классу Картрайт, если

$$
\int_{\mathbb{R}} \frac{\log _{+}|\varphi(x)|}{1+x^{2}} d x<\infty .
$$

Теорема 2. Для того чтобы матрииа де Бранжа $W$ была левым произведением Бляике-Потапова, необходимо и достаточно выполнения следующих условий:

1) $\liminf _{y \rightarrow \infty} \frac{\log \left|\Delta^{+}(i y)\right|}{y}=\liminf _{y \rightarrow-\infty} \frac{\log \left|\Delta^{-}(i y)\right|}{y}=0, \Delta^{ \pm}(z):=\alpha(z) \pm i \beta(z)$;

2) $\frac{\varphi(x)}{\Delta^{+}(x)} \notin L_{2}(\mathbb{R})$ для любой челой функции $\varphi \neq 0$ класса Картрайт и нулевой степени.

Из этой теоремы, а также из специальных интегральных представлений ц.ф.э.т. [6] выводится следуюший результат. 
ТЕОРема 3. Пусть выполнено условие 1) теоремы 2 и существует такой $A_{2}$-вес Макенхаупта $w^{2}$ на $\mathbb{R}[10]$, что

$$
\left|\Delta^{+}(x)\right|^{2} \ddot{\leqslant} w^{2}(x), \quad x \in \mathbb{R} .
$$

Тогда м.- $\phi$. . есть левое произведение Бляике-Потапова.

Доказательство следующей теоремы опирается на теорему 1 и свойства семейств рациональных дробей в весовых пространствах [6].

ТЕОрема 4. Если выполняется условие 1) теоремы 2 и существует такой





то м.-ф. W является правым произведением Бляике-Потапова.

СледСТВИЕ. Пусть м.- $\phi . W$ такова, что вес $\left|\Delta^{+}(x)\right|^{2}$ является $A_{2}$-весом Макенхаупта на $\mathbb{R}$. Тогда $W$ есть двустороннее произведение Бляике-Потапова $в$ том и только том случае, когда выполняется условие (9).

Оказьвается, что о м.-ф., рассмотренных в этом следствии, можно сказать существенно больше. Это объясняется тем, что отвечающие им операторы $B$ устроены достаточно просто.

Теорема 5. Пусть выполняется условие (9) $и\left|\Delta^{+}(x)\right|^{2}$ есть $A_{2}$-вес на $\mathbb{R}$. Тогда оператор $B$, определенный равенством (7), подобен ортогональной сумме $\left(B_{1}\right) \oplus\left(-B_{2}\right)$, әде $B_{1}, B_{2}$ - диссипативные операторы с одномерными мнимыми частями и полными семействами корневых подпространств.

Линейньй ограниченный оператор с полной системой корневых подпространств назьвается синтезируемым [10], [11], если сужение его на любое инвариантное подпространство также обладает полнотой корневых подпространств. Из теоремы 5 вытекает синтезируемость соответствующих операторов $B$, поскольку имеет место

Теорема 6. Ортогональная сумма $B_{1} \oplus\left(-B_{2}\right)$, где $B_{k}$ - диссипативный оператор с ядерной мнимой частью и полной системой корневых подпространств, $k=1,2$, синтезируема.

Рассмотрим некоторую факторизацию $W(z)=W_{1}(z) W_{2}(z)$, где $W_{k}, k=1,2$, как и $W$, суть совершенные матрицы де Бранжа без полюсов на вещественной оси и нормированы условием (3). Из теории характеристических м.-ф. следует [3], что каждому нетривиальному инвариантному подпространству оператора $B$ отвечает такая факторизация матрищы де Бранжа. Множитель $W_{1}$ (соответственно $W_{2}$ ) назьвается левым (соответственно правым) делителем $W$. Результат сформулированного ранее следствия усиливается следуюшим образом.

Теорема 7. Пусть м.- $\phi$. $W$ такова, что вес $\left|\Delta^{+}(x)\right|^{2}$ является $A_{2}$-весом Макенхаупта на $\mathbb{R}$ и выполняется условие (9). Тогда каждый правый (левый) делитель $W$ есть двустороннее произведение Бляике-Потапова.

И, наконец, сформулируем еще одну теорему, которая существенно дополняет результаты работы [12]. Напомним [13], что последовательность $U:=\left\{u_{k}\right\}_{1}^{\infty}, \operatorname{Im} u_{k}>0$, удовлетворяет условию Карлесона, если

$$
\inf _{k \geqslant 1} \prod_{j \neq k} \frac{\left|u_{k}-u_{j}\right|}{\left|u_{k}-\bar{u}_{j}\right|}>0 .
$$


Представим последовательность полюсов в виде $\Lambda=\Lambda_{+} \cup \Lambda_{-}, \Lambda_{ \pm}:=\Lambda \cap \mathbb{C}_{ \pm}$, и пусть $\bar{\Lambda}_{-}:=\left\{\bar{\mu}_{k}: \mu_{k} \in \Lambda_{-}\right\}$. Предполагается, что последовательности $\Lambda_{+}, \bar{\Lambda}_{-}$снова правильно занумерованы.

ТЕОрема 8. Пусть для матрицы де Бранжа $W$ выполняются условия теоремы 7. Если последовательности $\Lambda_{+} u \bar{\Lambda}_{-}$удовлетворяют условию Карлесона, то семейства функций

$$
\left\{k\left(z, \lambda_{n}\right): \lambda_{n} \in \Lambda\right\}, \quad\left\{\frac{\left(S^{\prime}\left(\lambda_{n}\right)\right)^{-1} S(z)}{z-\lambda_{n}}: \lambda_{n} \in \Lambda\right\}
$$

образуют взаимно биортогональные безусловные базисы пространства де Бранжа $\mathscr{H}(E)$, порождаемого матричей $W$.

3. Проиллюстрируем сфформулированные теоремы на одном простом примере. Пусть $S$ - сопутствующая пространству $\mathscr{H}(E)$ функция с $E(z)=e^{-i \omega z}, \omega>0$, корни которой простые и не лежат на $\mathbb{R}$. Проверяется, что $S$ - ц.ф.э.т., причем

$$
\int_{\mathbb{R}} \frac{|S(x)|^{2}}{1+x^{2}} d x<\infty, \quad h_{S}\left(\frac{\pi}{2}\right) \leqslant \omega, \quad h_{S}\left(-\frac{\pi}{2}\right) \leqslant \omega
$$

где $h_{S}(\varphi)$ - индикатор роста. Существует общая процедура [8] построения по заданным $E, S$ совершенной матрицы де Бранжа $W$ в вещественном представлении (6). В нашем частном случае

$$
W(z)=\frac{1}{S(z)}\left(\begin{array}{cc}
d(z) & c(z) \\
-\sin \omega z & \cos \omega z
\end{array}\right), \quad S(0)=1,
$$

где $c, d$ - вешественные аналитические функции, которые в $\mathbb{C}_{+}$задаются формулами

$$
\begin{gathered}
c(z)=\cos \omega z \Phi(z)-i S(z) S^{*}(z) e^{i \omega z}, \quad d(z)=-\sin \omega z \Phi(z)+S(z) S^{*}(z) e^{i \omega z}, \\
\Phi(z):=q+\frac{1}{\pi} \int_{-\infty}^{+\infty}\left(\frac{1}{x-z}-\frac{x}{1+x^{2}}\right)|S(x)|^{2} d x, \quad z \in \mathbb{C}_{+}, \quad q \in \mathbb{R}
\end{gathered}
$$

где $q$ определяется из равенства (3), т.е. из условия $(0)=0$. Если еще потребовать, чтобы детерминант матрицы (11) удовлетворял равенству (8), то сопутствующая функция $S$ однозначно будет определяться своими корнями:

$$
S(z)=\text { v.p. } \prod_{\lambda_{n} \in \Lambda}\left(1-\frac{z}{\lambda_{n}}\right) .
$$

В рассматриваемом случае $\mathscr{H}(E)$ является пространством Винера-Пэли с воспроизводящим ядром

$$
k(z, \lambda)=\frac{\sin \omega(z-\bar{\lambda})}{\pi(z-\bar{\lambda})} .
$$

Поэтому под действием преобразования ное с м.-ф. $W$ семейство $\left\{k\left(z, \lambda_{n}\right): \lambda_{n} \in \Lambda\right\}$ переходит в систему экспонент

$$
\left\{e^{i \bar{\lambda}_{n} t}: \lambda_{n} \in \Lambda\right\}, \quad \Lambda \cap \mathbb{R}=\varnothing
$$


которая в силу $(10)$ минимальна в $L_{2}(-\omega, \omega)$. Если м.-ф. $W$ есть левое произведение Бляшке-Потапова, то система (13) полна в $L_{2}(-\omega, \omega)$. Обратно, каждой полной и минимальной в $L_{2}(-\omega, \omega)$ системе $(13)$ по формуле $(12)$ отнесем функцию $S$, которая является ц.ф.э.т. и удовлетворяет условиям $(10)$, причем $h_{S}( \pm \pi / 2)=\omega[4]$. Поэтому $S$ сопутствует пространству $\mathscr{H}(E)$ с $E(z)=e^{-i \omega z}$. По приведенным выше формулам построим совершенную м.-ф. (11), которая будет левьм произведением Бляшке-Потапова. Таким образом, между полными и минимальными в $L_{2}(-\omega, \omega)$ системами $(13)$ и левыми произведениями Бляшке-Потапова вида (11) установлено биективное соответствие.

Поскольку в нашем примере $\Delta^{ \pm}(z)=e^{\mp i \omega z} S^{-1}(z)$, теоремы $1-4$ и теорему 8 можно переформулировать в виде утверждений о семействах экспонент и о биортогональных к ним системам, не упоминая вовсе о матрицах де Бранжа. Интересно, что даже в хорошо изученном случае семейств экспонент такие переформулировки теорем $1,3,4$ являются новыми. Теорема 2 оказалась равносильной критерию Левина полноты и минимальности семейств экспонент в пространстве $L_{2}(-\omega, \omega)[4]$. Если $\Lambda=\Lambda_{-}$, то теорема 8 впервые доказана Павловым [14], а в общем случае - это теорема Минкина о базисах из экспонент [15].

\section{СПИСОК ЦИТИРОВАННОЙ ЛИТЕРАТУРЫ}

[1] Потапов В.П. Мультипликативная структура $J$-нерастягивающих матриц-функций // Tp. MMO. 1955. T. 4. C. $125-236$.

[2] de Branges L. Hilbert spaces of entire functions: Prentice-Hall, 1968.

[3] Бродский М. С., Лившиц М. С. Спектральный анализ несамосопряженных операторов и промежуточные системы // УМН. 1958. Т. 13. № 1. С. 3-85.

[4] Левин Б.Я. Распределение корней целых функций. М.: Гостехиздат, 1956.

[5] Крейн М. Г. К теории целых функций экспоненциального типа // Изв. АН СССР. Сер. матем. 1947. Т. 11. С. 309-326.

[6] Губреев Г.М. Спектральная теория регулярных квазиэкспонент и регулярных $B$-представимых вектор-функций // Алгебра и анализ. 2000. Т. 12. № 6. С. 1-97.

[7] Arov D., Dym H. Matricial Nehari problems, $J$-inner matrix and the Muckenhoupt condition // J. Functional Analysis. 2001. V. 181. P. 227-299.

[8] Голинский Л.Б., Михайлова И.В. Гильбертовы пространства целых функций как объект $J$-теории. Препринт. Харьков: ФТИ НТ АН УССР, 1980.

[9] Сахнович Л. А. Методоператорных тождеств и задачи анализа // Алгебра и анализ. 1993. T. 5. №1. C. 3-80.

[10] Макрус А. С. Спектральный синтез вполне непрерывных операторов в банаховых пространствах // Функцион. анализ и его прилож. 2001. Т. 35. № 2. С. 74-78.

[11] Никольский Н. К. Современное состояние проблемы спектрального анализа-синтеза // Теория операторов в функциональных пространствах. Новосибирск: Наука, 1977.

[12] Губреев Г. М. Структура модельных вольтерровых операторов, биортогональные разложения и интерполяция в регулярных пространствах де Бранжа // Функцион. анализ и его прилож. 2001. Т. 35. № 2. С. 74-78.

[13] Гарнетт Дж. Ограниченные аналитические функции. М.: Мир, 1984.

[14] Павлов Б.С. Базисность систем экспонент и условие Макенхаупта // Докл. АН СССР. 1979. T. 247. №1. C. $37-40$.

[15] Минкин А. М. Отражение показателей и безусловные базисы из экспонент // Алгебра и анализ. 1991. Т. 3. № 5. С. 109-134.

(Губреев Г.М.) Южноукраинский государственный

Поступило

педагогический университет (г. Одесса)

28.03.2002

(Tарасенко A. А.) Universidad Autonoma del Estado de Hidalgo

Pachuca, Hidalgo, Mexico

E-mail: (Губреев Г.М.) varv@rambler.ru 